\title{
A Practical Approach to Asses Fatal Attacks in Enterprise Network to Identify Effective Mitigation Techniques
}

\author{
UMME SALSABIL ${ }^{1}$, M. TANSEER ALI ${ }^{2}$, MD. MANIRUL ISLAM ${ }^{3}$ \\ ${ }^{1}$ Graduate Student, Faculty of Engineering, American International University-Bangladesh \\ ${ }^{2}$ Assistant Professor, Faculty of Engineering, American International University-Bangladesh \\ ${ }^{3}$ Assistant Professor, Faculty of Science and IT, American International University-Bangladesh \\ E-mail: ${ }^{1}$ salsabil@aiub.edu, ${ }^{2}$ tanseer@aiub.edu, ${ }^{3}$ manirul@aiub.edu
}

\begin{abstract}
For any organization, having a secured network is the primary thing to reach their business requirements. A network is said to be secured when it can sustain from attacks, which may damage the whole network. Over the last few decades, internetworking has grown tremendously and lot of importance is given to secure the network. To develop a secure network, network administrators must have a good understanding of all attacks that are caused by an intruder and their mitigation techniques. This paper explores the most fatal attacks that might cause serious downtime to an enterprise network and examines practical approaches to understand the behavior of the attacks and devise effective mitigation techniques. It also describes the importance of security policies and how security policies are designed in real world.
\end{abstract}

Keywords: DoS Attack, ARP Spoofing, Evil Twin Attack, Man-in-the-middle Attack, DHCP Starvation.

\section{INTRODUCTION}

The Internet continues to grow exponentially. Personal, government, and business applications continue to multiply on the Internet, with immediate benefits to end users. However, these network-based applications and services can pose security risks to individuals and to the information resources of companies and governments. Information is an asset that must be protected. With the advent of new technologies, sophisticated attacks are increasing as well paralyzing enterprise network thus causing financial loss. According to statistical data, it is being observed that majority of the attacks are now being originated from inside network. So it has become more challenging to secure inside perimeter network as the traffic is not traversing the firewall and firewall by default trusts the inside network. The aim of this research is to assess the behavior of some of the fatal attacks using de-facto tools in an effort to identify effective and practical mitigation attacks. Choosing a particular mitigation technique for an attack has an impact on the overall performance of the network, because each attack has different ways for mitigation.

The attacks are carried out using both physical equipment and simulators. The data gathered is analyzed using industry standard data analysis tools to measure the efficacy of techniques that can significantly reduce network downtime.

\section{ATTACK ANALYSIS}

The following fatal attacks were being assessed:

\subsection{MAC Flooding Attack}

MAC flooding is a technique employed to compromise the security of network switches. Switches maintain a MAC Table that maps individual MAC addresses on the network to the physical ports on the switch. In a typical MAC flooding attack, a switch is fed many Ethernet frames, each containing different source MAC addresses, by the attacker. The intention is to consume the limited memory set aside in the switch to store the MAC address table. After launching a 
successful MAC flooding attack, a malicious user could then use a packet analyzer to capture sensitive data being transmitted between other computers, which would not be accessible were the switch operating normally.

To simulate the attack, we used Dsniffs 'macof' tool in Kali Linux environment in the attacker machine which generates random MAC addresses exhausting the switch's memory. It is capable of generating $155,000 \mathrm{MAC}$ entries on a switch per minute. But the question is, what happens if the switch is asked to process a constant stream of MAC addresses? In certain circumstances and on certain switches, this will cause the switch to go into a fail-safe mode, in which it basically turns into a hub. In other words, by overloading the switch, a hacker could have access to all the data passing through the switch.

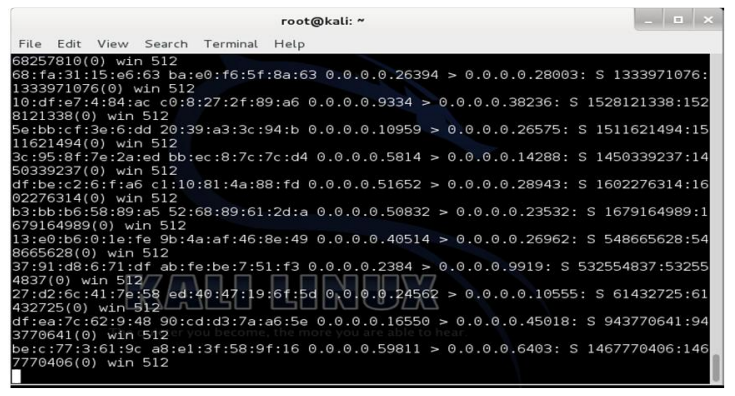

Fig. 1. MAC Flooding using macof

\subsection{DHCP Starvation Attack}

DHCP means Dynamic Host Configuration Protocol, where DHCP Server provides IP Address, Subnet Mask, Gateway Address and DNS Server Addresses. The following diagram illustrates how DHCP works.

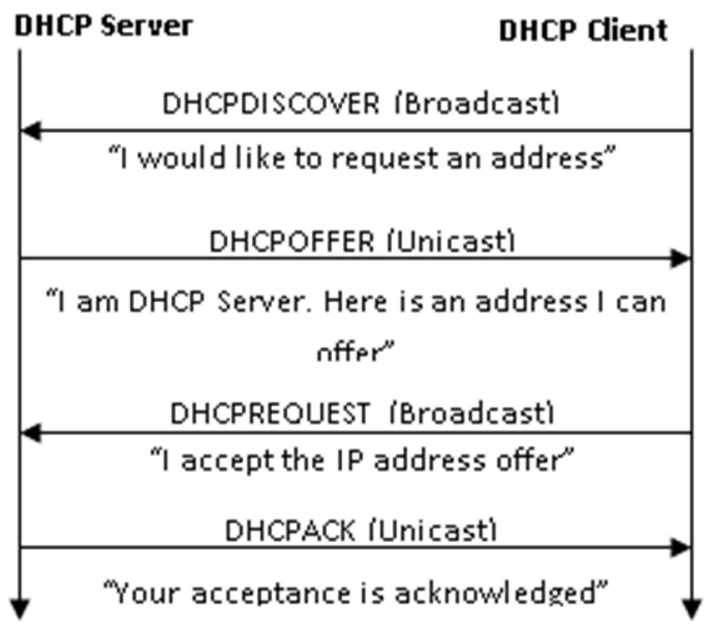

The intent of the DHCP Consumption Attack is for the Attacker to prevent hosts from gaining access to the network by denying them an IP address by consuming all of the available IP address in the DHCP Pool.

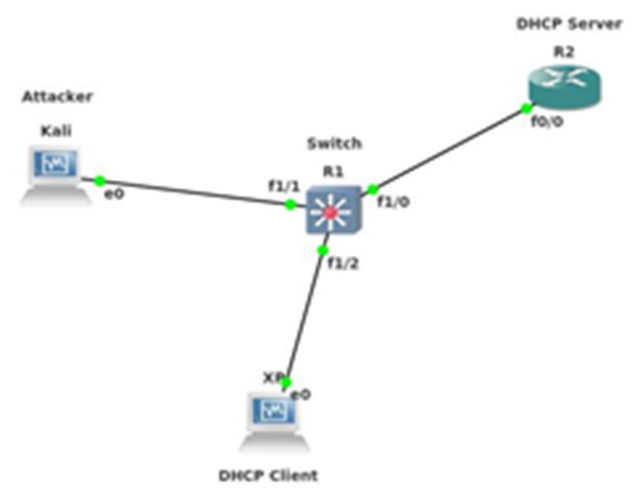

Fig. 3. DHCP Attack Test Scenario

To simulate real-world attack, we used Yersinia tool in Kali Linux environment and generated fake DHCP Discover messages from attacker machine. DHCP server address space was full within a while.

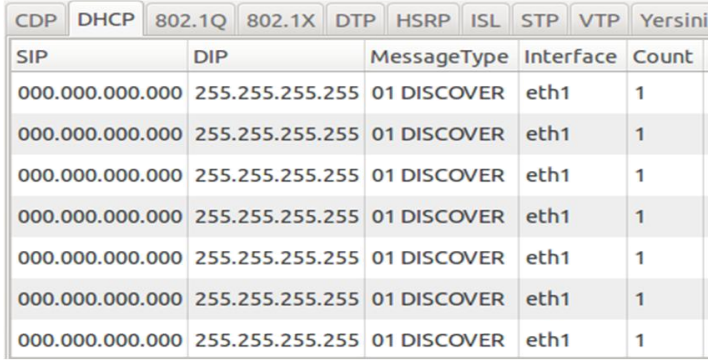

Fig. 4. DHCP Attack Using Yersinia

We used Wireshark tool to capture data from attacker machine to analyze the data for further investigation.

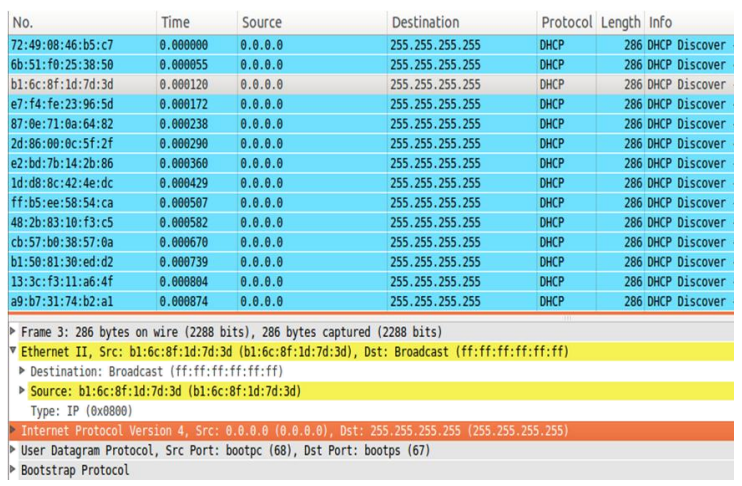

Fig. 5. Wireshark capture from attacker PC

Fig. 2. DHCP Operation 


\section{Wireshark Data Analysis}

$\begin{array}{lll}\text { Attack Ratio, PPS } & : & 35000 \text { (Avg.) } \\ \text { Attack Duration } & : & 1 \text { minute to } 5 \text { minute } \\ \text { Attack Source, } & : & \text { Random, Dynamic } \\ \text { MAC } & : & \text { DHCP Discover } \\ \begin{array}{l}\text { Attack Message } \\ \text { Type }\end{array} & : \begin{array}{l}\text { DHCP address pool } \\ \text { exhausted and } \\ \text { Attack Result }\end{array} & \begin{array}{l}\text { legitimate users will } \\ \text { not get IP address } \\ \text { from DHCP Server }\end{array}\end{array}$

\subsection{ARP Spoofing}

ARP stands for Address Resolution Protocol and it allow the network to translate IP addresses into MAC addresses. Basically, ARP works like this: When one host using IP on a LAN is trying to contact another it needs the MAC address of the host it is trying to contact. It first looks in its ARP cache to see if it already has the MAC address, but if not it broadcasts out an ARP request asking " who has this IP address I'm looking for?" If the host that has that IP address hears the ARP query it will respond with its own MAC address and a conversation can begin using IP. In common bus networks like Ethernet using a hub or $801.11 \mathrm{~b}$ all traffic can be seen by all hosts whose NICs are in promiscuous mode, but things are a bit different on switched networks. A switch looks at the data sent to it and tries to only forwards packets to its intended recipient based on MAC address. Switched networks are more secure and help speed up the network by only sending packets where they need to go. Using a program like Arpspoof, Ettercap or Cain we can lie to other machines on the local area network and tell them we have the IP they are looking for, thus funneling their traffic through us.

To simulate real-world attack, we used arpspoof tool in Kali Linux environment to redirect packets from a target host on the LAN intended for another host on the LAN by forging ARP replies.

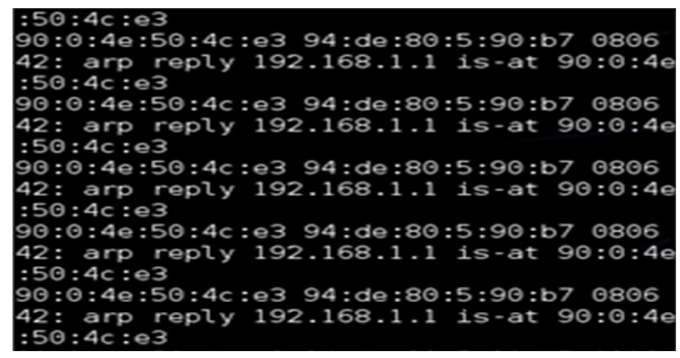

Fig. 6. ARP Spoofing
SSLStrip was being used to reroute encrypted HTTPS requests from network users to plaintext HTTP requests, effectively sniffing all credentials passed along the network via SSL. Finally, we used ettercap for credentials hijacking.

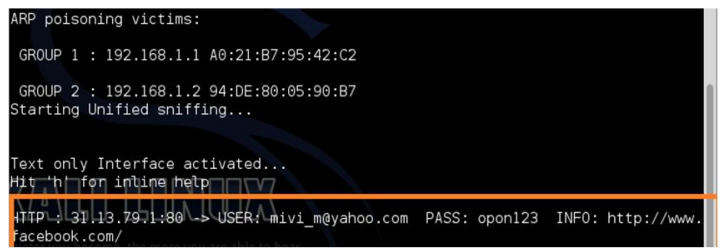

Fig. 7. Sniffed Data

In the victim machine, the only visible change is in ARP table. The attacker machine's MAC address replaces the gateway router's MAC address after ARP spoofing. From the Wireshark capture, we can clearly see that the MAC address of the destination host is that of the attacking machine.

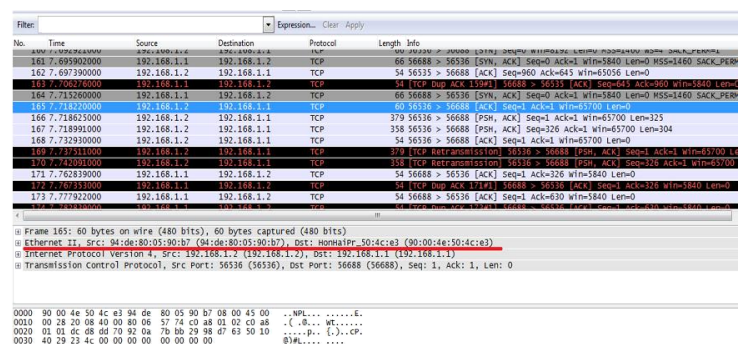

Fig. 8. Wireshark Capture of ARP Spoofing

In short, ARP Spoofing is the mother of most of the deadliest Man-in-the-Middle attacks [1].

\subsection{ICMP Flood Attack}

ICMP Flood attacks exploit the Internet Control Message Protocol (ICMP), which enables users to send an echo packet to a remote host to check whether it's alive. During a DDoS ICMP flood attack the agents send large volumes of ICMP_ECHO_ REPLY packets ("ping") to the victim. These packets request reply from the victim and this results in saturation of the bandwidth of the victim's network connection. During an ICMP flood attack the source IP address may be spoofed [4].

To simulate real-world ICMP flood attack, we used Hping3 tool to flood victim's machine with ICMP_ECHO_REPLY message. 


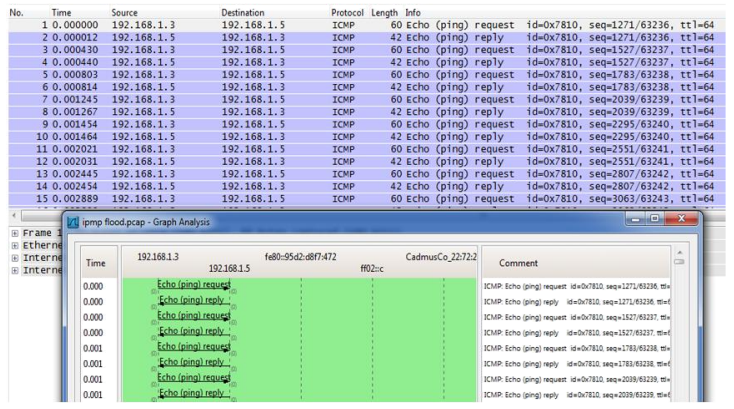

Fig. 9. Wireshark Capture of ICMP Flood Attack

\subsection{Wifi Jamming Attack}

Wi-Fi is increasingly becoming the preferred mode of internet connection all over the world. To access this type of connection, one must have a wireless adapter on their computer. Wi-Fi provides wireless connectivity by emitting frequencies between $2.4 \mathrm{GHz}$ to $5 \mathrm{GHz}$ based on the amount of data on the network. Since RF is essentially an open medium, jamming can be a huge problem for wireless networks. Jamming is one of many exploits used compromise the wireless environment. It works by denying service to authorized users as legitimate traffic is jammed by the overwhelming frequencies of illegitimate traffic. A knowledgeable attacker with advanced software like wirelessmon can detect and request connection to Hotspots and easily jam the $2.4 \mathrm{GHz}$ frequency in a way that drops the signal to a level where the wireless networks can no longer function.

To simulate real-world WiFi Jamming attack, we used airmon-ng to search for monitor interface and airodump-ng to get target network details e.g. ESSID, BSSID, and Channel Number. Then the attack can be launched using mdk3 or other wifijammer tool. The attack floods the wireless AP with unsolicited authentication messages and jams the wireless network.

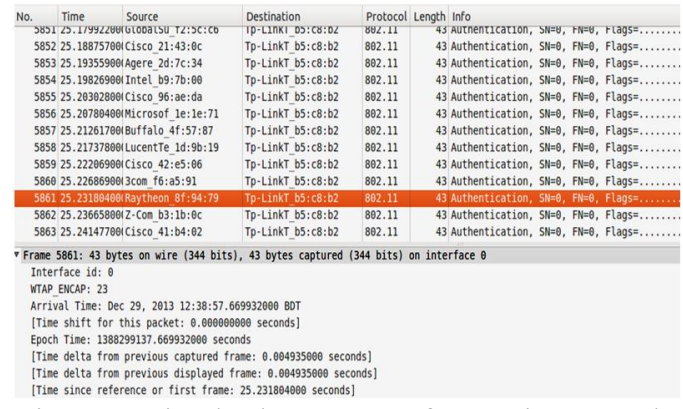

Fig. 10. Wireshark Capture of Jamming Attack

\section{Wireshark Data Analysis}

$\begin{array}{lll}\text { Attack Ratio } & : & 217 \text { PPS } \\ \text { Attack Type } & : & \text { Authentication Message } \\ & \text { from random spoofed } \\ & \text { sources } \\ \text { Attack Result } & : & \text { Jams the WiFi BSSID with } \\ & \text { unicast flood and other } \\ & \text { mobile stations would be } \\ & \text { disconnected from the } \\ & \text { network }\end{array}$

\subsection{Wifi Hacking}

WEP Wired Equivalent privacy uses weak 40 bit key \& short 24-bit initialization vectors to encrypt data. It was discovered that WEP could be cracked within minutes with standard off the shelf equipment. The reason for this weakness is the short IV (initialization vector) and the keys aren't changed, except by the user.

WEP uses the stream cipher RC4 for confidentiality and the CRC-32 checksum for integrity. The RC4 cipher stream is generated by a 40 or 64-bit RC4 key to encrypt and decrypt the data. There is also a 128 bit key that is used known as WEP2. The key is composed of a 24-bit IV (initialization vector) with a 40-bit WEP key. The user entered key is a 26 digit hexadecimal string where each character represents four bits of the key. The 26 digits represent 104 bit with addition of the 24-bit IV makes a 128-bit key.

The next security protocol, WPA (Wi-Fi Protected Access) was implemented because of the weaknesses in the WEP protocol. With APA there are two kinds of authentication types WPAEnterprise and WPA-Home. A good choice for small office and home use is WPA-PSK (PreShared Key) because it is simple to setup and is compatible with many types of hardware. WPAPSK uses 8 to 63 ASCII or 64 hex digit character pass-phrase created by the user and entered in a client. The stronger this key, the stronger the security is because weak keys are subject to password cracking.

A stronger form of WPA released in 2004 is known as WPA2. The advantage of WPA2 is that it provides stronger encryption with the use of AES (Advanced Encryption Standard) which may be a requirement for some government or corporate users. All WPA2 that are Wi-Fi certified are backward compatible with WPA. WPA and WPA2 both use "fresh" sessions using a unique encryption keys for each client which are specific to that client. 


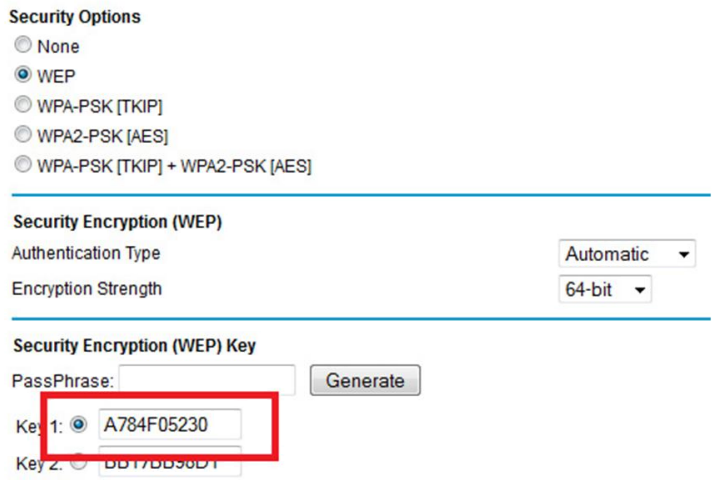

Fig. 11. WEP Passphrase into WiFi Router

To simulate real-world attack, we used wifite tool to crack WEP passphrase. Wifite automatically puts a wireless interface into monitor mode and starts scanning for the nearby wireless networks. After selecting the ESSID, wifite automatically starts processing and find the passphrase.

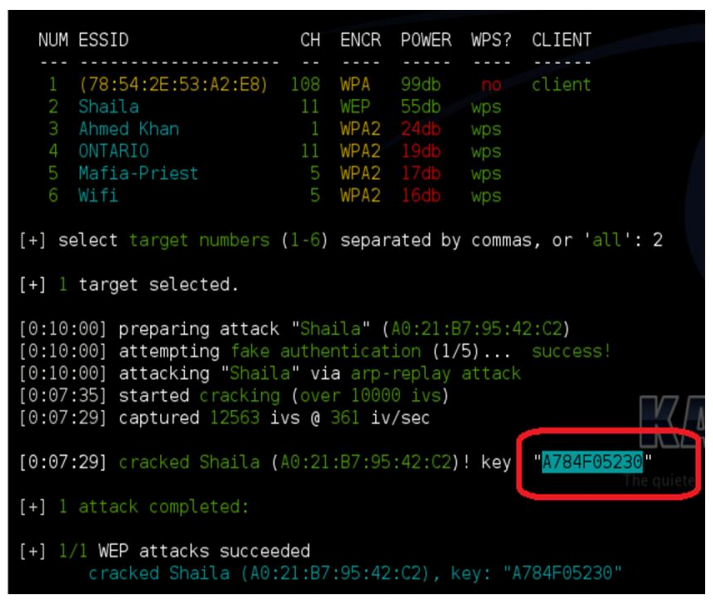

Fig. 12. WEP Passphrase found in Wifite

\subsection{WIRELESS EVIL TWIN ATTACK}

Anywhere public Wi-Fi is available is an opportunity for an attacker to use that insecure hot spot to attack unsuspecting victims. One specific Wi-Fi hot spot attack called an "Evil Twin" access point can impersonate any genuine Wi-Fi hot spot. Attackers will make sure their evil twin AP is just like the free hot spot network, and users are then duped when connecting to an evil twin AP and the attacker can execute numerous attacks to take advantage of the unaware victim.

A typical evil twin attack is illustrated in the graphic below.

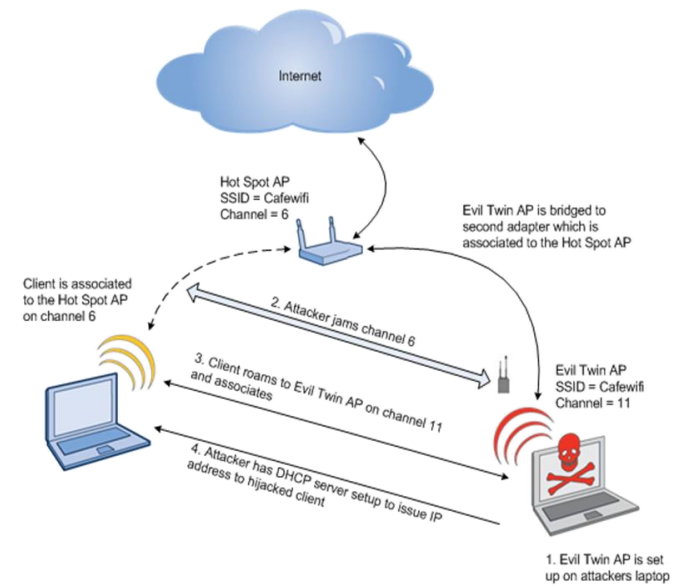

Fig. 13. Evil Twin Attack Scenario

To simulate real-world attack, we used airmon-ng to start wireless interface into monitor mode. Then we used easy-creds to create fake AP. Ettercap, SSLStrip, URL Snarf, DSniff were used to sniff user credentials.

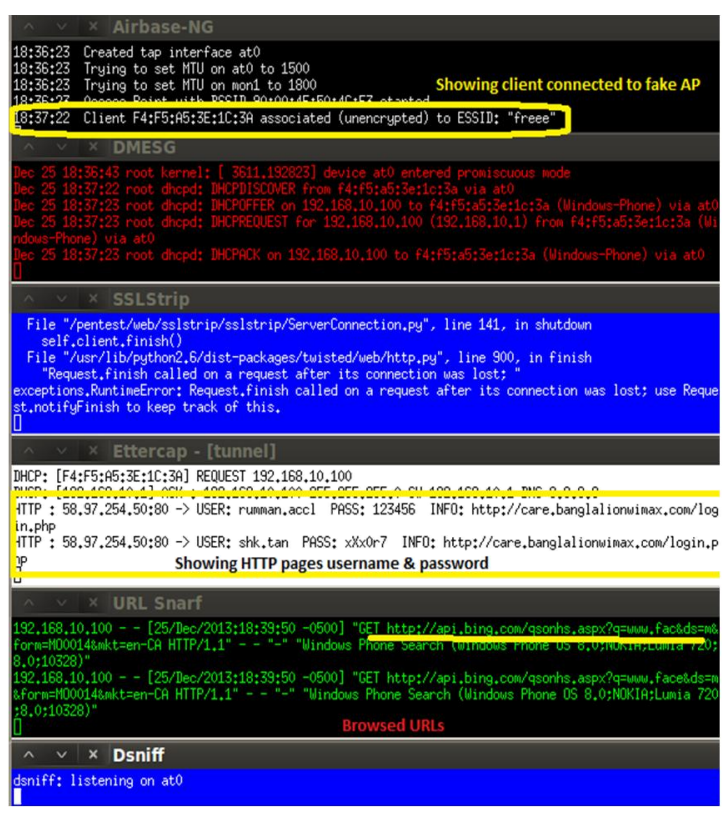

Fig. 14. Sniffing User Data Connected to fake AP

\section{MITIGATION TECHNIQUES}

Choosing a particular mitigation technique for an attack has an impact on the overall performance of the network, because each attack has different ways for mitigation. We used real-world scenarios to 
initiate the attacks so that we can come up with practical and effective mitigation techniques. Suggested mitigation techniques follow:

\subsection{MAC Flooding Attack}

Mitigation of the CAM table-overflow attack can be achieved by configuring port security on the switch. This will allow MAC addresses to be specified on a particular switch port, or alternatively, specify the maximum number of MAC addresses that the switch port can learn. If an invalid MAC address is detected on the switch port, the port can be shut down, or the MAC address can be blocked.

Sticky MAC addresses are also a viable solution when implementing the mean to mitigate CAM Table Overflows. The MAC address will be learned when the first MAC address attempts to connect to the port and will be written to the running configuration. Statically a MAC address could be in on the port also.

Packet capture from attacker machine state that, attack ratio is random, means source and destination is random. As a result, switch mac address-table flooded with random mac addresses.

As a mitigation technique, we can use port security at switch port for limited number of mac addresses and also can bind the mac address to the switch port. We can also use storm-control in switch port to mitigate the attack.

\section{Pseudocode:}

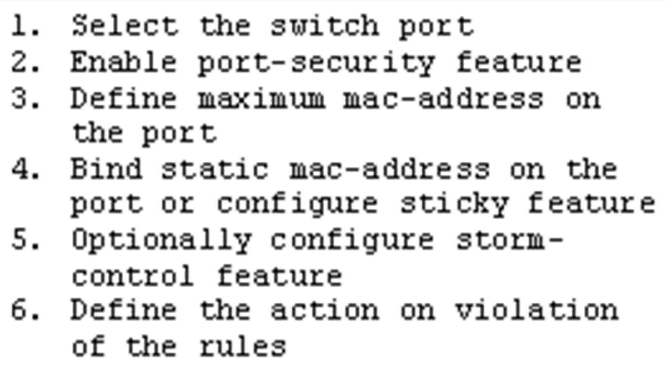

1. Select the switch port

2. Enable port-security feature

3. Define maximum mac-address on the port

4. Bind static mac-address on the port or configure sticky feature

5. Optionally configure stormcontrol feature

6. Define the action on violation of the rules

\subsection{DHCP Starvation Attack}

DHCP Starvation Attack can be mitigated using storm-control feature in switch port.

But before we enable storm-control in switch port, we need to identify normal traffic pattern and traffic rate in every switch port and compare the normal traffic with attacker machine traffic.

According to attacker machine, traffic rate is 35000 pps during broadcast DHCP Discover message. Let the normal traffic rate be 100 to 10000 pps. So a threshold value of 30000 pps would do the trick. This is the most cost-effective solution.

\section{Pseudocode:}

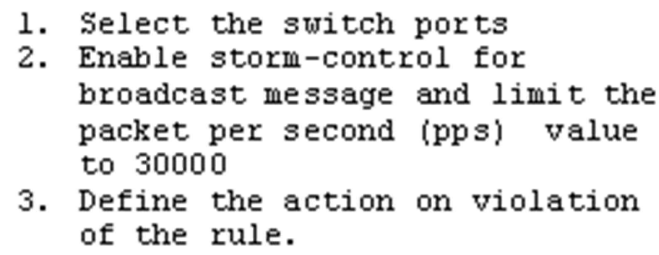

3. Define the action on violation of the rule.

\subsection{ARP spoofing}

ARP Spoofing can be prevented in several effective ways.

\subsubsection{Static ARP table}

Static Address Resolution Protocol (ARP) entry is a permanent entry in your ARP cache. One reason you may want to add static ARP entries is if you have two hosts that communicate with each other constantly throughout the day; by adding static ARP entries for both systems in each other's ARP cache, you reduce some network overhead, in the form of ARP requests and ARP replies.

\subsubsection{Arpwatch}

Arpwatch is an open source computer software program that helps you to monitor Ethernet traffic activity (like Changing IP and MAC Addresses) on your network and maintains a database of ethernet/ip address pairings. It produces a log of noticed pairing of IP and MAC addresses information along with a timestamps, so you can carefully watch when the pairing activity appeared on the network. It also has the option to send reports via email to a network administrator when a pairing added or changed.

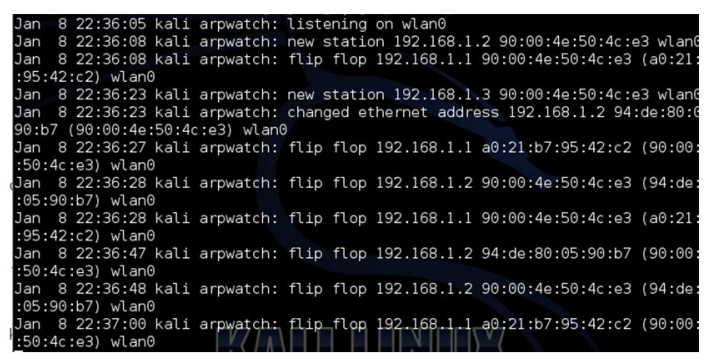

Fig. 15. Arpwatch Detecting ARP Spoof 


\subsubsection{Dynamic ARP Inspection (DAI)}

ARP inspection prevents malicious users from impersonating other hosts or routers (known as ARP spoofing). By default, all ARP packets are allowed through the security appliance. You can control the flow of ARP packets by enabling ARP inspection.

When you enable ARP inspection, the security appliance compares the MAC address, IP address, and source interface in all ARP packets to static entries in the ARP table, and takes the following actions:

If the IP address, MAC address, and source interface match an ARP entry, the packet is passed through. If there is a mismatch between the MAC address, the IP address, or the interface, then the security appliance drops the packet.

The attacker, however, sends another ARP response to the host with the attacker MAC address instead of the router MAC address. The attacker can now intercept all the host traffic before forwarding it on to the router. ARP inspection ensures that an attacker cannot send an ARP response with the attacker MAC address, so long as the correct MAC address and the associated IP address are in the static ARP table.

Another important feature of DAI is that it implements a configurable rate-limit function that controls the number of incoming ARP packets. This function is particularly important because all validation checks are performed by the CPU, and without a rate-limiter, there could be a DoS condition.

\subsection{ICMP Flood Attack}

To defend against ICMP Flood Attack, iptables script can be applied as below:

\begin{tabular}{|ll|}
\hline 1 & iptables -N icmp_flood \\
& iptables -A INPUT -p icmp -j \\
& icmp_flood \\
3 & iptables -A icmp_flood -m limit -- \\
& limit 1/s --limit-burst 3 -j RETURN \\
4 & iptables -A icmp_flood -j DROP \\
\hline
\end{tabular}

After iptables rules is applied, if the attacker is sending ICMP Echo Request packets continuously, victim's machine will not respond by sending ICMP Echo Reply packets as all the packets are being dropped by the firewall.

If DDoS attack is not that excessive, an appropriate configuration of the operating system and affected service could help to counteract the attack. Linux kernel parameters that enable to modify the behavior when faced with certain circumstances. Some of these parameters can be found in /etc/sysctl.conf.

tcp_syncookies: protects you against Syn Flood attacks. The way it works is as follows: when the syn segment request queue completes, the kernel responds with a syn-ack segment as normal, but creates a special, encrypted sequence number that represents the source and target IP, the port and the timestamp of the received packet. Activate syn cookies with:

sysctl -w net.ipv4.tcp_syncookies=1

ignore broadcasts: ICMP (echo request) packets are sent to a broadcast address in Smurf attacks with a false IP source. This false IP is the target of the attack, as it receives multiple echo reply response packets as a result of the broadcast packet sent by the attacker. One way of deactivating the ICMP echo-broadcast requests is by activating the following option:

sysctl -w net.ipv4.icmp_echo_ignore_ broadcasts $=1$

rp_filter: Known also as source route verification, it has the same purpose as Unicast RPF (Reverse Path Forwarding) 14 and uses Cisco routers. It is used to check that the packets that enter via an interface are accessible based on the source address, making it possible to detect IP Spoofing:

sysctl -w net.ipv4.conf.all.rp_

filter $=1$

For attacks that are performed by programs like LOIC, it is also possible to implement measures using iptables and hashlimit modules to limit the number of packets that you want a particular service to accept.

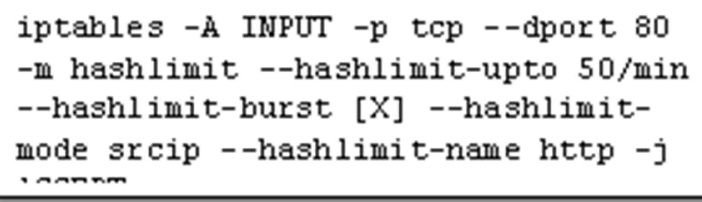


The clauses hashlimit-burst and hashlimit-upto set the maximum size of the bucket and the number of IP packets that limit the connections to port 80.

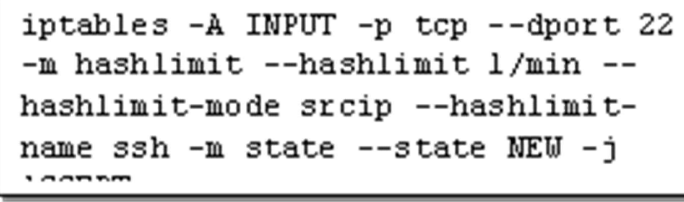

You can also take steps to resist numerous forceful attacks at services such as ssh, ftp, etc. by limiting the number of IPs allowed per minute.

Regardless of the measures adopted in the operating system, it is recommended that public services such as web services, FTP, DNS, etc located in a DMZ (Demilitarized Zone) are made secure separate to the rest. For example, in the case of Apache it would be very useful to give it modules such as mod_evasive, mod_antiloris, mod_security, mod_reqtimeout or similar to help fight against a great variety of DDoS attacks against this platform.

\subsection{WiFi Jamming Attack}

Jamming attack detection is the prerequisite of jamming attack mitigation method. It is so important that the operation of jamming attack mitigation cannot be performed unless the jamming attack has been detected. It is a big challenge to detect the jammers because there are different kinds of jammers and even the same jammer can switch between different jamming models or jamming powers. There are also lots of network conditions, such as low throughput, normal communication, congestion, and so on, which have similarity with the jammed network, making it difficult to distinguish the jamming situations from legitimate ones. The jamming attacks should also be differentiated from the special circumstances, such as system power off, operating system hung up, antenna problems, communicating distance and so on, which may also lead to the similar results as the jamming attacking. For example, if the attack occurred on an RF corresponding to channel 1, the access point should switch to channel 6 or 11 in order to avoid the attack. However, selecting a different channel does not always eliminate the issue of interference. An experienced attacker will often use all available channels in the attack.

The nature of the Wi-Fi jamming attack relies on the discovery of ESSID and BSSID of the Access Point or Wireless Router. So the best way to mitigate Wi-Fi jamming attack is to disable SSID broadcast. The attacker machine will not find the ESSID and BSSID and channel number for attack.

\subsection{WiFi Hacking}

The Mitigation of Wi-Fi Hacking requires strict implementation of security policies throughout the network.

\subsubsection{Security Policy}

Wireless LAN implementation in a large corporation without any security policies will put the corporation at serious risk. In fact, all organizations should have a security policy in regards to wireless LAN infrastructure in place before reaching the deployment stage.

i. Before implementing a wireless LAN and during the planning phase, you need to know who are your users and where are they seated in order to ensure the access point signal is adequate to cover the necessary areas.

ii. Scanning and detecting for rogue access points on the corporate network regularly is a must.

iii. The default management passwords and SSIDs on access points should be changed prior to installing them into corporate network. Strong passwords should be used when changing the passwords with at least 8 characters in length.

iv. Educate users to be aware of security \& Enforcing that employees should not rogue access points into the corporate network.

\subsubsection{Network Level Security}

\section{i. Isolation of Wireless LAN}

The wireless LAN should be implemented on another network separate from your internal wired LAN. This means that the access points should be installed on a separate network with a firewall in placed between the wireless network and the wired corporate network.

\section{ii. $\quad$ Securing Wireless LAN with VPN Solution}

As discussed earlier, there are many security vulnerabilities found with WEP. It is recommended to include Virtual Private Network (VPN) solution into your wireless LAN to ensure secure wireless connections. 
iii. Authentication and Authorization via RADIUS Before allowing a wireless client to connect and access to the corporate private network, it is a must to validate or authenticate that client. This can be achieved by using $802.1 \mathrm{X}$ authentication on a remote authentication dialin user service (RADIUS) server.

iv. Handling the SSIDS

The default SSIDs on the access points should be changed prior to installation into the corporate network. Disable the broadcast SSID option though attacker can sniff the SSID by using Kismet software.

v. Handling the SSIDS

Access Control via MAC Addresses and IP Addresses

Access points can be configured to filter MAC addresses to control users connecting to your corporate wireless network. This means those users with valid MAC addresses that had been configured on access points will be allowed connectivity to the wireless network.

\subsection{Wireless Evil Twin Attack}

In most existing techniques the detection of the attack is performed by the network not by the users. One of the original ways of doing so was by the manual detection using software like Netstumbler, by the administration of the network.

AirDefense uses a combination of radiofrequency sensors jointly with an intrusion detection server, capturing, processing and correlating network events trying to find APs with unknown fingerprints.

Wavelink is mobile device management that features a software installed on each mobile client to detect connectivity faults. Among other things the client software reports to a central server any AP seen and its location which is than matched with a list of legal Aps.

Other solutions like RIPPs use different approaches to detect wireless traffic in wired networks to detect the existing of illegal APs.

However, most of these solutions suffer from some, or all, of the following problems:

- They do require complete coverage of the network otherwise rogue APs may go undetected.
- They may flag a normal AP as rogue. For instance, the access point of a nearby coffee shop.

- $\quad$ They do not work for rogue APs that possess authentication

- They may access unauthorized networks in the process of testing all the available APs in the vicinity.

- And finally, they are ineffective in reacting to short time attacks. For instance, if an attack is detected on some area of an airport how do we go and alert the users; it may be too late.

To date, Evil Twin attack can most effectively be mitigated through Multi-hop Detection.

\section{CONCLUSION}

In this research, we tried to describe several ways of analyzing traffic depending on the circumstances and the available means, as well as providing examples of some common attacks used on local area networks to mitigate or at least moderate the impact that these generate on the performance of your network. There are several areas of potential future work in this area that could be explored. This study attempted to test as many types of common enterprise configurations as possible but left out several that are in use or will continue to grow in the future Although this study attempted to record data as accurately as possible it could be done even more accurately if an automated process was developed to track throughput over a period of time and report the results.

\section{REFERENCES}

[1] Edward W. Felten, Dirk Balfanz, Drew Dean, and Dan S. Wallach, "Web Spoofing: An Internet Con Game", Technical Report Department of Computer Science, Princeton University, February 1997, pp. 540-96.

[2] Radosavac, S., Crdenas, A.A., Baras, J.S., Moustakides, G.V, "Detecting IEEE 802.11 MAC layer misbehavior in ad hoc networks: Robust strategies against individual and colluding attackers", Journal of Computer Security 15 2007, pp.103-128. 
[3] Hayoung Oh, Inshil Doh, Kijoon Chae, "Attack Classification Based on Data Mining Technique and its Application for Reliable Medical Sensor Communication", International Journal of Computer Science and Applications, Vol. 6, No. 3, 2009, pp. 20-32.

[4] J. Markovic, J. Martin, and L. Reiher, "A Taxonomy of DDoS Attack and DDoS Defense Mechanisms", ACM SigComm Computer Communication Review, Vol. 34, No. 2, 2004, pp. 39-53.

[5] Kong, H.S., Zhang, M.Q., Tang, J. and Luo, C.Y, "The Research of Simulation for Network Security Based on System Dynamics", Information Engineering University, Institute of Electronic Technology, Zhengzhou, China, IAS, vol. 2, 2009, pp 145-148.

[6] A. Hussain, J. Heidemann, and C. Papadopoulos, "A framework for classifying denial of service attacks", In Proceedings of the Conference on Applications, Technologies, Architectures, and Protocols for Computer Communications, SIGCOMM, 2003, pp. 99110.

[7] K. Argyraki and D. R. Cheriton, "Active internet traffic filtering: real-time response to denial-of-service attacks", In Proceedings of the annual conference on USENIX Annual Technical Conference, 2005, pp. 10-10.

[8] V. Gulisano, R. Jim'enez-Peris, M. PatĩnoMart'inez, and P. Valduriez. Streamcloud, "A large scale data streaming system", In International Conference on Distributed Computing Systems, June 2010, pp. 126-137.

[9] Al-Saadoon, G, Al-Bayatti, H, "A Comparison of Trojan horse Virus Behavior in Linux and Windows Operating Systems", World of Computer Science and Information Technology jornal, Vol. 1, No. 3, 2011, pp. 56-62.

[10] Thimbleby,H., Anderson,S. and Cairns, A framework for Modelling Trojan horse $\mathrm{s}$ and Computer Virus Infection, Computer Journal, Vol. 41, No. 7, 1998, pp. 444-458.

[11]Liu,y., Zhang,l. Liang,j. Qu,s. Ni,z, "Detecting Trojan horses based on system behavior using machine learning method", Machine Learning and Cybernetics conference IEEE, vol 2, 2010 , pp. $855-860$.

[12] Tang, Sh, "The detection of Trojan horse based on the data mining", Fuzzy Systems and Knowledge Discovery International Conference IEEE, vol. 1, 2009, pp. 311-314.

[13]B.N. Singh, Bhim Singh, Ambrish Chandra, and Kamal Al-Haddad, "Digital Implementation of an Advanced Static VAR
Compensator for Voltage Profile Improvement, Power Factor Correction and Balancing of Unbalanced Reactive Loads", Electric Power Energy Research, Vol. 54, No. 2, 2000, pp. 101-111.

[14]Z. Yang, A. C. Champion, B. Gu, X. Bai, and D. Xuan, "Link-layer protection in 802.11i WLANS with dummy authentication," Wisec, 2009.

\section{AUTHOR PROFILES:}

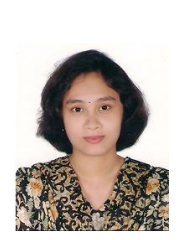

Umme Salsabil received the degree in Bachelor of Science in Electrical and Electronics Engineering from American International UniversityBangladesh in 2012. She is a research student under Faculty of Engineering at AIUB pursuing Master of Science in Electrical and Electronics Engineering majoring in Communication Engineering. Currently, she is working as an Instructor under Continuing Education Center at American International University-Bangladesh. Her interests are in wired and wireless LAN security.

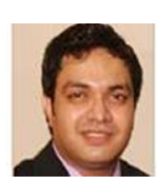

M. Tanseer Ali received his $\mathrm{PhD}$ degree in Electrical and Electronics engineering from University of Greenwich, UK. Currently, he is serving as an Assistant Professor under Faculty of Engineering at American International University-Bangladesh. His research interests include Telecommunication Engineering and Power System Dynamics.

Md. Manirul Islam received his B.Sc. in Computer Engineering from University of Baguio and MSc. in IT from Saint Louis University. Currently, he is serving as an Assistant Professor under Faculty of Science and Information Technology and Director, Continuing Education Center at American International University-Bangladesh. His research interests include Network Intrusion Detection and Wireless Sensor Networks. 